\title{
Automatic liver and tumor segmentation based on deep learning and globally optimized refinement
}

\author{
HONG Yuan ${ }^{1} \quad$ MAO Xiong-wei ${ }^{2,3, \dagger}$ \\ HUI Qing-lei ${ }^{1}$ \\ OUYANG Xiao-ping ${ }^{4}$ \\ PENG Zhi-yi ${ }^{2, *}$ \\ KONG De-xing ${ }^{1, *}$
}

\begin{abstract}
Automatic segmentation of the liver and hepatic lesions from abdominal 3D computed tomography (CT) images is fundamental tasks in computer-assisted liver surgery planning. However, due to complex backgrounds, ambiguous boundaries, heterogeneous appearances and highly varied shapes of the liver, accurate liver segmentation and tumor detection are stil1 challenging problems. To address these difficulties, we propose an automatic segmentation framework based on 3D U-net with dense connections and globally optimized refinement. Firstly, a deep U-net architecture with dense connections is trained to learn the probability map of the liver. Then the probability map goes into the following refinement step as the initial surface and prior shape. The segmentation of liver tumor is based on the similar network architecture with the help of segmentation results of liver. In order to reduce the influence of the surrounding tissues with the similar intensity and texture behavior with the tumor region, during the training procedure, $I \times$ liver $_{\text {label }}$ is the input of the network for the segmentation of liver tumor. By doing this, the accuracy of segmentation can be improved.The proposed method is fully automatic without any user interaction. Both qualitative and quantitative results reveal that the proposed approach is efficient and accurate for liver volume estimation in clinical application. The high correlation between the automatic and manual references shows that the proposed method can be good enough to replace the time-consuming and non-reproducible manual segmentation method.
\end{abstract}

\section{$\S 1 \quad$ Introduction}

The prevention and treatment of liver diseases is a major focus of current research for clinical diagnosis $[1,2]$. Liver cancer has been reported as the second most frequent cause of cancer

\footnotetext{
Received: 2021-02-18. Revised: 2021-03-24.

MR Subject Classification: 68T07, 68U10.

Keywords: liver segmentation, tumor segmentation, CT, deep learning.

Digital Object Identifier(DOI): https://doi.org/10.1007/s11766-021-4376-3.

Supported by the National Natural Science Foundation of China(12090020,12090025) and Zhejiang Provincial Natural Science Foundation of China(LSD19H180005).

${ }^{\dagger}$ Equal contribution.

* Corresponding author.

(c)The Author(s) 2021.
} 
death in men and the sixth leading cause of cancer death in women. Indeed, about 750,000 people were diagnosed with liver cancer and nearly 696,000 people died from this disease worldwide in 2008 [3]. Accurate detection and delineation of liver and tumor is a crucial prerequisite for many clinical treatments, such as liver resection, transplantation and radiotherapy treatment planning. In the currently clinical applications, the delineation and detection of liver and tumor are always done manual on each slice by experts.But manual segmentation is subjective, poorly reproducible and time-consuming. Therefore, it is necessary to develop automatic method to accelerate and facilitate diagnosis, therapy planning and monitoring. However, the segmentation of liver and tumor are still challenges due to the following reasons. Firstly, low-contrast between liver and tumor, liver and surrounding regions makes both the liver and tumor boundaries weak/fuzzy and difficult to detect. Secondly, there are different types of contrast levels of tumors (hyper-/hypo-intense tumors), which always lead to complicated intensity distributions and heterogeneous texture appearance. Thirdly, abnormalities in tissues (metastasectomie), size and varying amount of lesions also increase the difficulty of segmentation.

In the past few decades, several state-of-the-art algorithms, including region-based methods, active-contour models, graph cut and machine learning, have been proposed to segment the liver and tumor. Region-based method including region growing [4], region splitting and merging [5], and watershed methods [6]. Liu et al. [7] used a gradient vector flow(GVF) [8] based active contour model for the segmentation CT liver images. Massoptier et al. [9] proposed a statistical model-based approach to distinguish hepatic tissue from other abdominal organs, then the statistical based model was incorporated into the GVF model for the segmentation of both liver and tumor. Shaikhli et al. [10] developed a level set method based on the sparse representation of global and local image information for the segmentation of liver from 3D CT volume images. Wang et al. [11] defined a shapeCintensity prior level set model to delineate liver boundaries that incorporates both the probabilistic atlas and probability map constrains. Compared with the previously methods, graph cut-based methods, which is the extension of the classic graph cut proposed by Boykov et al. [12,13] are more popular in liver segmentation. Li et al. [14] proposed a likelihood and local constraint level set model for liver tumor detection. Peng et al. [15] combined intensity, regional appearance, and surface smoothness within a variational framework to deal with fuzzy boundaries and heterogeneous backgrounds. Furthermore, seed constraints, both in the foreground and background, were used in the constrained convex variational model in [16]. However, due to the speed and robustness to the noise and heterogeneous in CT images, these methods are not widely applied in clinics. Hence, more methods are still needed to overcome these weaknesses.

Recently, deep learning models, which can learn a hierarchy of features by building high-level features from low level ones, have received researchers attention. Deep learning has been applied to a wide variety of problems and has surpassed the previous state-of-the-art performance, which motivates us to apply this approach to fully automatic liver tumor segmentation in CT. A. Ben-Cohen et al. [17] explored an FCN for the task of liver segmentation and livermetastasis detection in CT examinations. Christ et al. [18] presented a method to automatically segment liver and lesions in CT abdominal images using cascaded fully convolutional neural 
networks (CFCNs) and dense3D conditional random fields (CRFs). Lu et al. [19] proposed a method (called 3D CNN-GC) that combined 3D fully CNNs and graph cuts to achieve automatic segmentation in CT images. The trained CNN generated a probability map of the liver and then the learned information was integrated into the image data penalty term of graph cuts.

In this paper, we proposed a method to automatically segment liver and tumors in CT abdomen images using 3D U-net with dense connections and graph cut-based globally optimized refinement. The proposed segmentation framework is based on 3D U-net architecture with dense connections and globally optimized refinement. Firstly, a deep U-net architecture with dense connections is trained to learn the probability maps of the liver. Then the probability maps go into the following refinement step as the initial surfaces and prior shapes. The dense connections between layers can encourage feature reuse and reduces the number of parameters while maintaining good performance. By concatenating feature maps from coarse to fine layers, the network allows capturing multi-scale contextual information. Segmentation of liver tumor is based on the similar network architecture with the help of segmentation results of liver. In order to reduce the influence of the surrounding tissues with the similar intensity and texture behavior with the tumor region, during the training procedure, $I \times$ liver $_{\text {label }}$ is the input of the network for the segmentation of liver tumor. By doing this, the accuracy of segmentation can be improved. A data set of 1161 images with background labeled by experienced radiologists is used for training and 100 images are used to evaluate the algorithm. The proposed algorithm achieves a mean Dice similarity coefficient of $73.6 \%$ on test image data set. Experimental results show that the proposed method can be served as an alternatives to replace the time-consuming and non reproducible manual segmentation method.

The rest of the paper is organized as follows. In the next four sections, Section 2 reviews graph cut method and U-net network. And we describe the proposed method in detail.Section 3 illustrates the results and provides a comparative discussion of the proposed algorithm. We conclude the paper in Section 4.

\section{$\S 2$ Materials and Methods}

In this section, we present the imaging data used in this study and the proposed automatic liver segmentation framework. In the training stage, deeply dense-connected U-net architecture is trained using labeled CT images. In the testing stage, given a test image, a probability map of the liver is learned by the trained network. Then, the probability map is thresholded to provide both initialization and shape prior for the following refinement segmentation step. In the refinement segmentation step, the liver was segmented based on set of prior information. The energy functional of this step incorporates initial seeds location, the liver probability map, intensity distribution and region appearances. Finally, the energy functional is minimized using a global optimization-based approach to propagate the initial surface to the optimal position. 


\subsection{Materials}

In our experiments, totally 1161 volume images from 294 patients with liver tumor were used for training and 100 images for testing. Common CT liver images have four phases, including plain scan, arterial phase, venous phase and portal phase. For the screening reason, the plain scan is the common used. In such way, most of the volume images are the plain scan images. Among all the images, 279 patients have all the four phase images and 15 patients have three phase images. 25 patients with 4 phased images, totally 100 volume images are used as testing data set. All the images are from the First Affiliated Hospital of Zhejiang University. The images have axial dimensions of $512 \times 512$ with slice numbers varying from 32 to 86 , and the slice thickness is $5 \mathrm{~mm}$. The corresponding segmentation labels (both liver and tumor) were obtained by trained technicians with our home-developed semiautomatic liver segmentation tool, and then the results were approved and revised by experienced radiologists. Examples of the images used in the paper is shown in Fig.1.

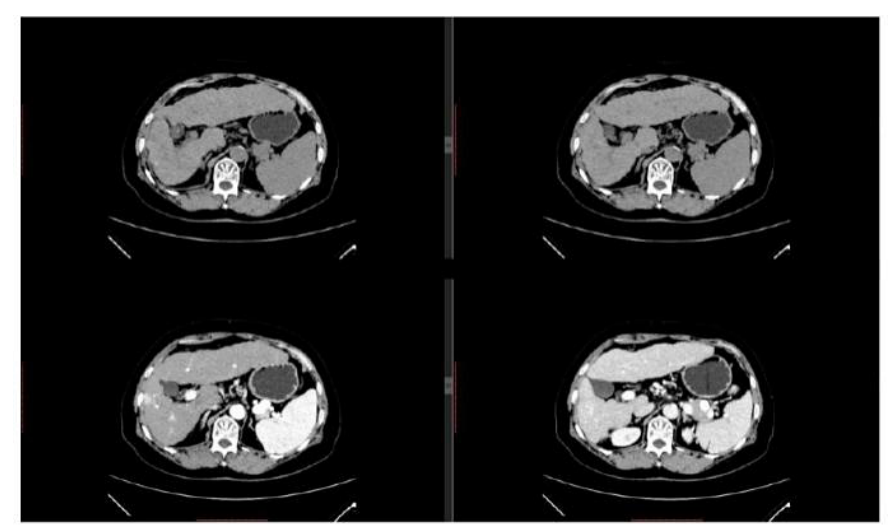

Figure 1. Examples of the images with four phases used in the paper.

\subsection{Preprocessing}

Preprocessing was carried out in a slice-wise fashion. Firstly, the images were normalized using the information in the corresponding header files by using the following equation:

$$
\bar{x}=\left\{\begin{array}{cl}
1, & x>\max \\
\frac{x-\min }{\max -\min }, & \min \leq x \leq \max \\
0, & x<\min
\end{array}\right.
$$

where $\min =w c-\frac{w w}{2}, \max =w c+\frac{w w}{2}, w c$ denotes the window level and $w w$ means the window width. Then the total slice number is unified to 40 . For the volume images with slicer number smaller than 40 , we put several $512 \times 512$ slices with intensity value of 0 at both the beginning and the end of the image to make sure the slice number is 40 and the volume image is in the middle of the new generate image. For the images with slicer number larger than 40 and smaller than 80 , we deleted the slices in the beginning and the end of the image. For the slice number 
larger than 80 , we use the inter layer sampling along the $\mathrm{Z}$ axis, then unify the slice number to 40. In the proposed algorithm, the input image is $512 \times 512 \times 40$.

\subsection{Proposed U-net architecture with dense connections}

The architecture of the proposed network is shown in Fig.2. For the liver segmentation, the input images $(512 \times 512 \times 40)$ are firstly entering a convolution layer with a kernel size of $(7 \times 7 \times 3)$ and stride of $(2,2,2)$, then a convolution layer with kernel size of $(5 \times 5 \times 3)$ and stride of $(2,2,1)$ is applied. Then a max-pooling layer with size of $(3 \times 3 \times 3)$ and stride of $(2,2,2)$ is applied to down-sample the images into quarter sized $(64 \times 64 \times 10)$. The quarter sized image is the input of the dense block. The proposed network contains 4 dense blocks, each dense block contains 4, 6, 16 and 8 dense layers respectively. The growth rate is set to 32 . Each dense block is connected by the transition layer. The transition layer contains convolution layer with kernel sizes of $(1 \times 1 \times 1)$ or $(2 \times 2 \times 2)$ and stride of $(2,2,2)$ or $(2,2,1)$, and average pooling layers. The transition layer act as down sampling and descending the number of channels. The size of the output image of the fourth dense block is $(8 \times 8 \times 5)$. In order to achieve the probability maps with the same resolution with the original images, up-sampling is then applied on the outputs of the 4th dense block, then the up sampled features are concatenate with the outputs with the same size from previous layer. All the activation function is chose to ReLU. We use sigmoid function to activate the last convolutional layer to gain the corresponding probability map. The final output of the network is $(512 \times 512 \times 40 \times 2), 2$ channels are background and liver respectively. The details of the network architecture are shown in Table1.

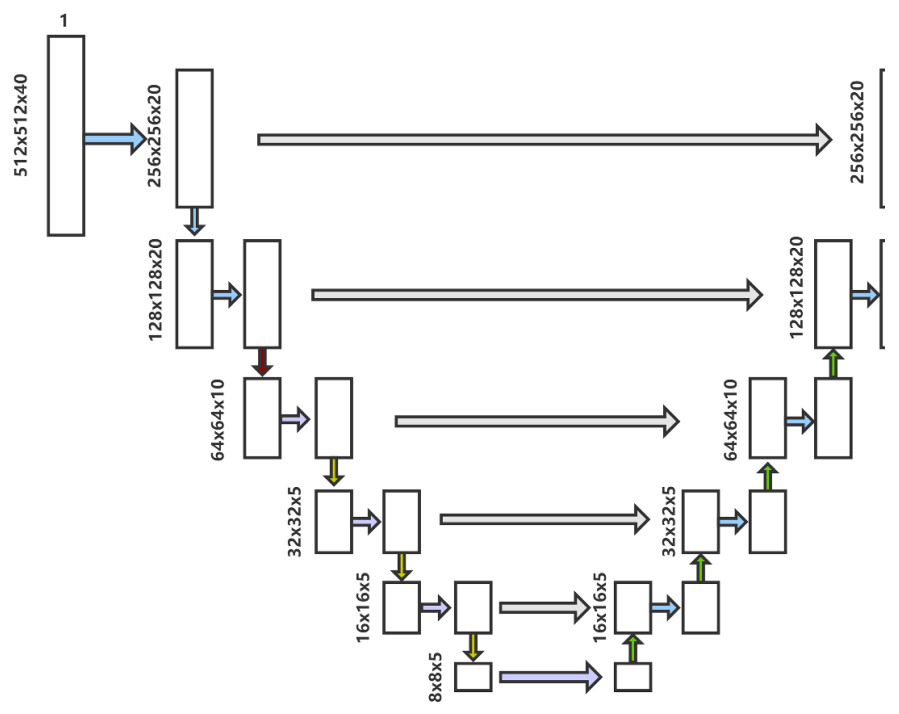

Figure 2. U-net architecture with dense connections for the segmentation of liver and tumor.

For the tumor segmentation, we adopt the same network architecture of the live segmenta- 
Table 1. Details of the proposed architecture for the segmentation of liver.

\begin{tabular}{|c|c|c|}
\hline & |3D Dense U-NET & Feature size \\
\hline Input & & $512 \times 512 \times 40$ \\
\hline Convolution1 & $\mid 7 \times 7 \times 3$,stride: $(2,2,2), 96$ & $256 \times 256 \times 20$ \\
\hline Convolution2 & $\mid 5 \times 5 \times 3$,stride: $(2,2,1), 128$ & $128 \times 128 \times 20$ \\
\hline Max pooling & $\mid 3 \times 3 \times 3$,stride: $(2,2,2), 96$ & $64 \times 64 \times 10$ \\
\hline Dense block1 & $\mid(1 \times 1 \times 1$ conv $+3 \times 3 \times 3$ conv $) \times 4$ & $64 \times 64 \times 10$ \\
\hline Transition1 & $\mid 1 \times 1 \times 1$ conv $+2 \times 2 \times 2$ AP, stride $2(2,2,2) \mid$ & $32 \times 32 \times 5$ \\
\hline Dense block2 & $\mid(1 \times 1 \times 1$ conv $+3 \times 3 \times 3$ conv $) \times 6$ & $32 \times 32 \times 5$ \\
\hline Transition2 & $\mid 1 \times 1 \times 1$ conv $+2 \times 2 \times 2$ AP, stride $2(2,2,1) \mid$ & $16 \times 16 \times 5$ \\
\hline Dense block3 & $\mid(1 \times 1 \times 1$ conv $+3 \times 3 \times 3$ conv $) \times 16$ & $16 \times 16 \times 5$ \\
\hline Transition3 & $\mid 1 \times 1 \times 1$ conv $+2 \times 2 \times 2$ AP, stride $2(2,2,1) \mid$ & $8 \times 8 \times 5$ \\
\hline Dense block 4 & $\mid(1 \times 1 \times 1$ conv $+3 \times 3 \times 3$ conv $) \times 8$ & $8 \times 8 \times 5$ \\
\hline US1+Dense block3 & stride: $(2,2,1)$ & $16 \times 16 \times 5$ \\
\hline US2+Dense block2 & | stride: $(2,2,1)$ & $32 \times 32 \times 5$ \\
\hline US3+Dense block1 & stride: $(2,2,2)$ & $64 \times 64 \times 10$ \\
\hline US4+Convolution2 & | stride: $(2,2,2)$ & $128 \times 128 \times 20$ \\
\hline US5+Convolution1 & | stride: $(2,2,2)$ & $256 \times 256 \times 20$ \\
\hline US6 & | stride: $(2,2,2)$ & $512 \times 512 \times 40$ \\
\hline Convolution3 & $\mid 5 \times 5 \times 3$,stride: $(1,1,1), 2$ & $512 \times 512 \times 40$ \\
\hline
\end{tabular}


tion except the first two convolutional layers. The inputs are $I \times$ liver $_{\text {label }}$, where the liver $_{\text {label }}$ is the segmentation results of the liver. By doing this, we can focus on the region inside liver and prevent miss-locating the tumor area to the outside of the live region. The input images $(512 \times 512 \times 40)$ are firstly entering a convolution layer with a kernel size of $(3 \times 3 \times 3)$ and stride of $(2,2,2)$, then a convolution layer with kernel size of $(3 \times 3 \times 3)$ and stride of $(2,2,1)$ is applied. Since the tumor region is small compared with the liver region, in order to achieve a smaller receptive field, the kernel size of first two convolutional layers are smaller than the ones for liver segmentation. The details of the network architecture for the segmentation of liver tumor are shown in Table 2.

Table 2. The details of the network architecture for the segmentation of liver tumor.

\begin{tabular}{|c|c|c|}
\hline & 3D Dense U-NET & Feature size \\
\hline Input & & $512 \times 512 \times 40$ \\
\hline Convolution1 & $3 \times 3 \times 3$, stride: $(2,2,2), 96$ & $256 \times 256 \times 20$ \\
\hline Convolution2 & $3 \times 3 \times 3$, stride $:(2,2,1), 128$ & $128 \times 128 \times 20$ \\
\hline Max pooling & $3 \times 3 \times 3$, stride: $(2,2,2), 96$ & $64 \times 64 \times 10$ \\
\hline Dense block1 & $\mid(1 \times 1 \times 1$ conv $+3 \times 3 \times 3$ conv $) \times 4$ & $64 \times 64 \times 10$ \\
\hline Transition1 & $1 \times 1 \times 1$ conv $+2 \times 2 \times 2$ AP, stride $2(2,2,2)$ & $32 \times 32 \times 5$ \\
\hline Dense block2 & $\mid(1 \times 1 \times 1$ conv $+3 \times 3 \times 3$ conv $) \times 6$ & $32 \times 32 \times 5$ \\
\hline Transition2 & $1 \times 1 \times 1$ conv $+2 \times 2 \times 2$ AP, stride $2(2,2,1)$ & $16 \times 16 \times 5$ \\
\hline Dense block3 & $\mid(1 \times 1 \times 1$ conv $+3 \times 3 \times 3$ conv $) \times 16$ & $16 \times 16 \times 5$ \\
\hline Transition3 & $\mid 1 \times 1 \times 1 \mathrm{conv}+2 \times 2 \times 2$ AP, stride $2(2,2,1)$ & $8 \times 8 \times 5$ \\
\hline Dense block 4 & $\mid(1 \times 1 \times 1$ conv $+3 \times 3 \times 3$ conv $) \times 8$ & $8 \times 8 \times 5$ \\
\hline US1+Dense block3 & stride: $(2,2,1)$ & $16 \times 16 \times 5$ \\
\hline US2+Dense block2 & stride: $(2,2,1)$ & $32 \times 32 \times 5$ \\
\hline US3+Dense block1 & stride: $(2,2,2)$ & $64 \times 64 \times 10$ \\
\hline US4+Convolution2 & stride: $(2,2,2)$ & $128 \times 128 \times 20$ \\
\hline US5+Convolution1 & stride: $(2,2,2)$ & $256 \times 256 \times 20$ \\
\hline US6 & stride: $(2,2,2)$ & $512 \times 512 \times 40$ \\
\hline Convolution3 & $5 \times 5 \times 3$, stride: $(1,1,1), 2$ & $512 \times 512 \times 40$ \\
\hline
\end{tabular}

For the liver segmentation, the model uses the Adam optimizer and the binary cross-entropy as the loss function.

$$
C=-\frac{1}{M} \sum_{i}^{M}\left[y_{i} \log \widetilde{y}_{i}+\left(1-y_{i}\right) \log \left(1-\widetilde{y}_{i}\right)\right]
$$

where $y_{i}$ denotes the ground truth of voxel $i, \widetilde{y}_{i}$ denotes the output prediction probability of the network, $M$ is the number of voxels in one image $(512 \times 512 \times 40)$. 
The initial learning rate $l_{0}$ was 0.0001 and decayed according to the equation $l_{0} \times 0.5^{\left(\left\lfloor\frac{1+n}{5}\right\rfloor\right)}$, where $\lfloor\cdot\rfloor$ denotes the rounded down operator, $n$ is the training period.Almost all tumors are significantly smaller than livers, so for the tumor segmentation, Adam optimizer is adopted and the weighted categorical cross-entropy is used as loss function.

$$
C=-\frac{1}{M} \sum_{i}^{M}\left[(1-w) y_{i} \log \widetilde{y}_{i}+w\left(1-y_{i}\right) \log \left(1-\widetilde{y}_{i}\right)\right]
$$

Here $y_{i}$ denotes the ground truth of voxel $i, \widetilde{y}_{i}$ means the output of the network, $w$ is the weight balancing tumor and background. $w=\frac{V_{\text {tumor }}}{V_{\text {total }}}$, where $V_{\text {tumor }}$ is the volume of tumor, $V_{\text {total }}$ is the total volume of image. In order to accelerate the convergence of the network and gain more precisely training results, we set the learning rate as: $l_{r}=l_{0} \times\left(1-\frac{n}{N+1}\right)^{0.9}$, $l_{0}=0.0001, N$ is the number of epochs which is set to 40 .

\subsection{Globally optimized refinement}

The outputs of the networks are not precisely enough if we directly threshold the probability maps of the liver and the tumor. We need post processing to get more smooth, precise and connective segmentation results. In this paper, we simply threshold the probability maps of the liver and the tumor as the initial foreground. Than we adopt graph-cut based method to refine the segmentation results. In this section, a novel energy function that integrates region statistics and shape prior constraint is proposed. The energy functional is defined as:

$$
E(l)=\lambda E_{D}(l)+E_{B}(l)
$$

where $l$ denotes the labels of all voxels, $E_{D}(l)$ and $E_{B}(l)$ represent the region term energy and boundary term energy respectively, and $\lambda$ is the weight balancing them. The boundary term energy is defined as:

$$
E_{B}(l)=\int_{\partial \Omega} \frac{1}{1+\delta|\nabla I(x)|^{2}} d x
$$

where $\Omega$ is one entire volume image, $\nabla I(x)$ represents the square sum of gradients in three directions. The region term energy is defined as:

$$
E_{D}(l)=\int_{\Omega}\left[\max (R(x), 0) l_{x}+\max (-R(x), 0)\left(1-l_{x}\right)\right] d x
$$

where $l_{x}$ is the label of voxel $\mathrm{x}, R$ denotes the region term consisting of voxel intensity, voxel intensity cumulative histogram, and the output probability map of the proposed network. The region term is defined as:

$$
R(x)=\alpha \frac{\left(I(x)-I_{\max }\right)\left(I(x)-I_{\min }\right)}{\left(I_{\max }-I_{\min }\right)^{2}}+\beta \mid H \text { ist }\left._{\text {local }}(x)\right|_{L_{1}}-\gamma(P(x)-T)
$$

Here, $I(x)$ denotes the intensity value of voxel $x$ in the image $I$ and $I_{\min }$ and $I_{\max }$ denote the value of $5 \%$ and $95 \%$ of the initial foreground intensity histogram respectively. $\mid$ Hist $\left._{\text {local }}(x)\right|_{L_{1}}$ represents the $L 1$ norm between the cumulative histogram of the initial foreground region and cumulative histogram of the $(5 \times 5 \times 5)$ neighborhood region centered at $x$, which is defined as: 


$$
\left|\operatorname{Hist}_{\text {local }}(x)\right|_{L_{1}}=\sum_{j}^{K}\left|H i s t_{\text {initial }}^{j}-H i s t_{\text {local }}^{j}\right|
$$

where $H i s t_{\text {initial }}^{j}$ and $H i s t_{\text {local }}^{j}$ indicate the $j$-th value of cumulative histogram of initial foreground and neighborhood region centered at $x, K=125 . P(x)$ denotes the output probability of the proposed network. $T$ represents the threshold value, where we chose 0.5 for liver segmentation and 0.75 for tumor segmentation. In our experiment we set $\alpha=0.2, \beta=$ $I_{\max }-I_{\min }, \gamma=4 \beta$.

\section{$\S 3 \quad$ Results}

The proposed model is trained using Keras and Tensorflow python libraries on a NVIDIA GTX 1080 Ti GPU. The total training time is about 26 hours each for liver and tumor segmentation. The post globally optimized refinement is implemented using $\mathrm{C}++$, and the average runtime for one image globally optimized refinement is about 17 seconds. Figure 3 shows the loss and accuracy of training and test dataset of tumor segmentation network. As we can see the result of test data set is more volatile than that achieved by training data set, but still has convergent tendency.

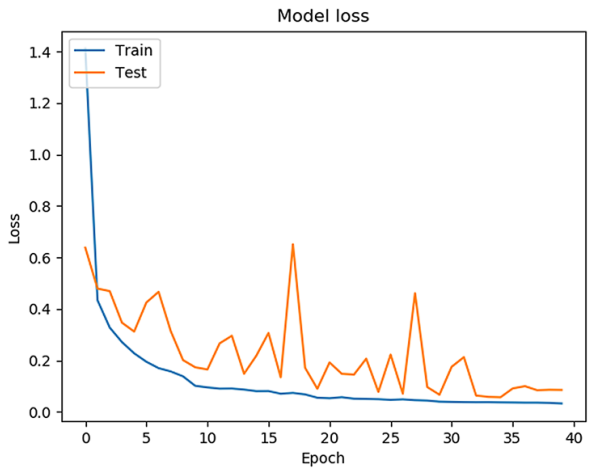

(a)

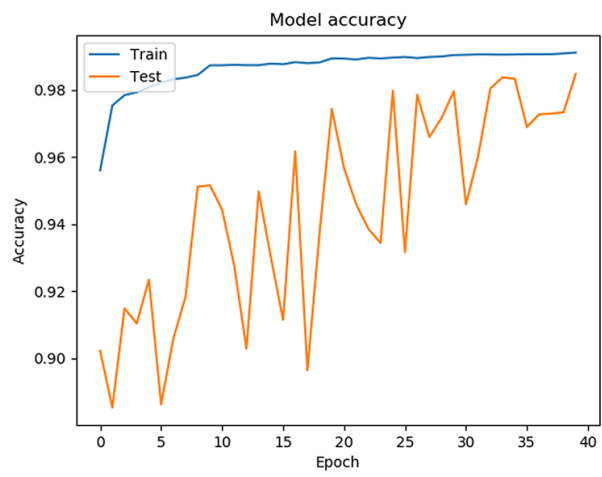

(b)

Figure 3. (a)Training loss and (b)Training accuracy of the tumor segmentation network.

To evaluate the liver and tumor segmentation result of our proposed model, we calculate dice similarity coefficient(DSC), root mean square symmetric surface distance(RMSD), mean square symmetric surface distance(MSD), average symmetric surface distance(ASD), and relative volume difference(RVD). The five mean evaluation metrics of 100 test voxel image segmented by our framework are summarized in table3. The better segmentation result of liver is due its relatively larger size and regular shape. Our network and post refinement model are based on $3 \mathrm{D}$, for clarity, we demonstrate some segmentation result of slices in one test voxel image. As shown in Fig 4, the green contours are manually segmentation results by radiologists, the red 
contours indicate the final liver segmentation result of our framework.

Table 3. Liver and tumor segmentation results on testing cases.

\begin{tabular}{c|c|c|c|c|c|}
\hline & DSC & RMSD & ASD & MSD & RVD \\
\hline liver & 97.07 & 1.98 & 0.174 & 47.22 & $0.32 \%$ \\
\hline tumor & 73.6 & 1.82 & 21.4 & 81.02 & $6.49 \%$ \\
\hline
\end{tabular}

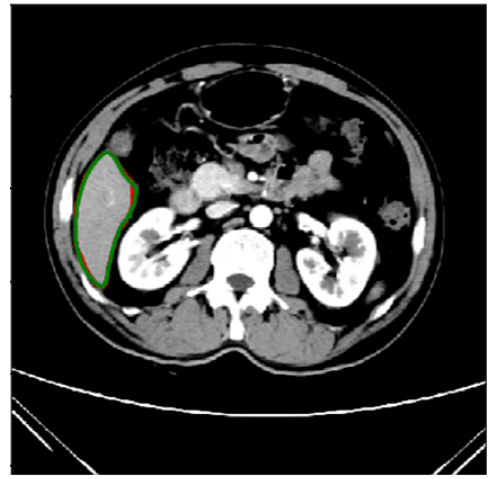

(a)

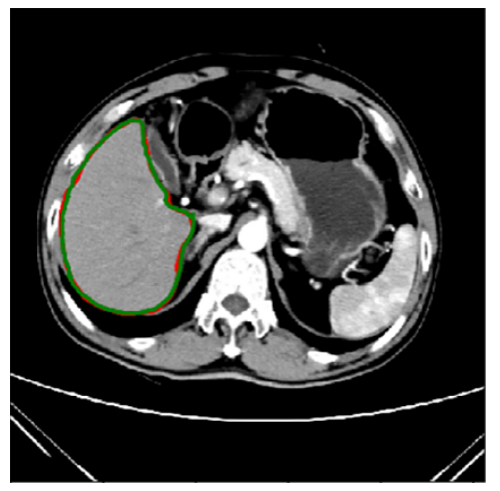

(c)

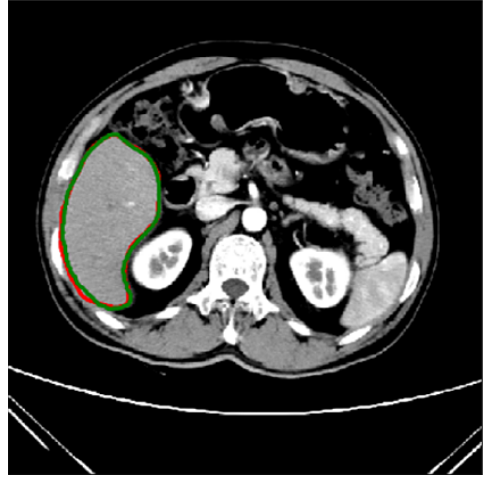

(b)

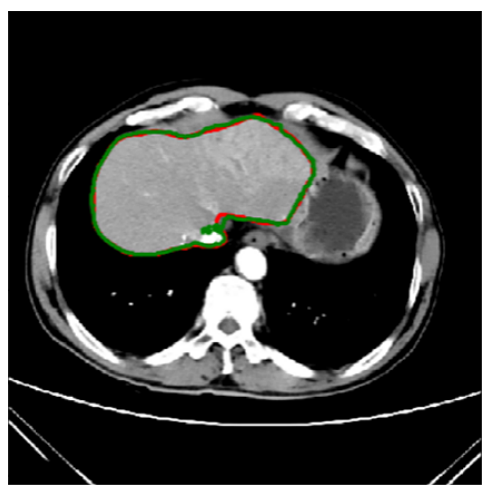

(d)

Figure 4. Example of liver segmentation result of a testing image.

The tumor segmentation result of one test voxel image is shown in Fig 5. The green contour on the left is manually delineated by radiologists, yellow contour is obtained by thresholding of probability map and shown on the right. We can see simply thresholding the probability map cannot get accurate segmentation. The red contour indicates the final tumor segmentation result of our framework, which demonstrate our post globally optimized refinement process can effectively solve the under segmentation problem caused by threshoding. Also the 3D reconstruction result in Fig.6 illustrates our final result which is recognized by radiologists. 

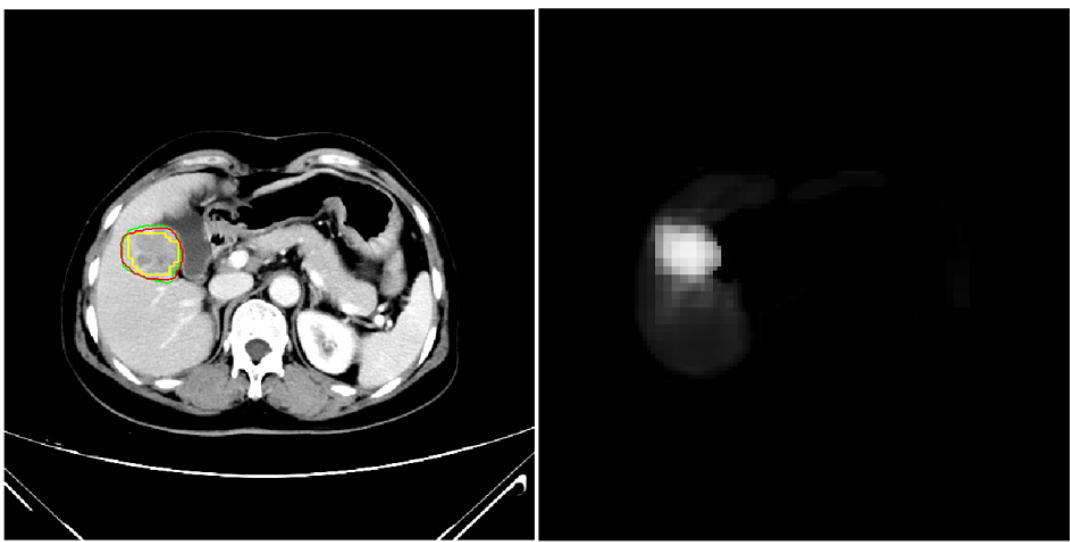

Figure 5. Example of tumor segmentation result of a testing image.

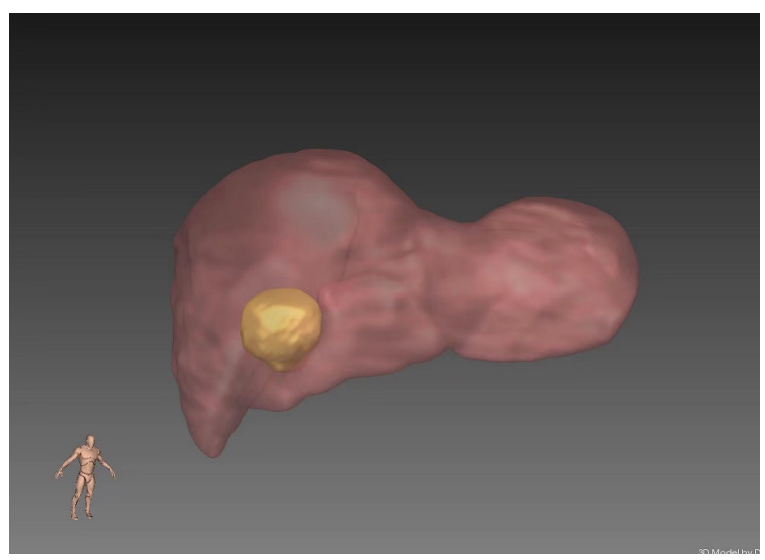

Figure 6. 3D rendering of tumor segmentation.

\section{$\S 4$ Conclusion}

In this study, we explored 3D network based on U-net with dense connections for automatic liver segmentation in abdominal CT images. Specifically, a 3D model was trained for automatic liver location. The learned liver probability map was then integrated into the graph cut energy function for further segmentation refinement. Meanwhile, based on the result of liver segmentation, we applied the same framework to liver tumor segmentation. Liver tumor probability map was obtained to generate an initial segmentation. The learned probability map was then integrated into the graph cut energy function for further segmentation refinement. The main advantage of our method is that it does not require any user interaction for initialization. The high correlation between our segmentation and manual reference indicated that the proposed method has the clinical applicability for hepatic volume estimation. In the future, we will apply our method to more liver and tumor data, and other medical image segmentation tasks, such as kidney and spleen segmentation. 
Open Access This article is licensed under a Creative Commons Attribution 4.0 International License, which permits use, sharing, adaptation, distribution and reproduction in any medium or format, as long as you give appropriate credit to the original author(s) and the source, provide a link to the Creative Commons licence, and indicate if changes were made. The images or other third party material in this article are included in the articles Creative Commons licence, unless indicated otherwise in a credit line to the material. If material is not included in the articles Creative Commons licence and your intended use is not permitted by statutory regulation or exceeds the permitted use, you will need to obtain permission directly from the copyright holder. To view a copy of this licence, visit http://creativecomm ons.org/licenses/by/4.0/.

\section{References}

[1] A S Haugen, S Eirik, S K Almeland, N Sevdalis, et al. Effect of the World Health Organization Checklist on Patient Outcomes: A Stepped Wedge Cluster Randomized Controlled Trial, Annals of Surgery, 2015, 261(5): 821-828.

[2] S M Macédo, A G Talita, J D Feltenberger, S H S Santos. The role of renin-angiotensin system modulation on treatment and prevention of liver diseases, Peptides, 2014, 62: 189-196.

[3] L A Torre, F Bray, R L Siegel, J Ferlay, et al. Global cancer statistics, CA: A Cancer Journal for Clinicians, 2015, 65(2): 87-108.

[4] L Ruskó, G Bekes, M Fidrich. Automatic segmentation of the liver from multi- and single-phase contrast-enhanced CT images, Medical Image Analysis, 2009, 13(6): 871-882.

[5] R Susomboon, D Raicu, J Furst, D Channin. Automatic Single-Organ Segmentation in Computed Tomography Images, Proceedings of the 6th IEEE International Conference on Data Mining, 2006: 1081-1086.

[6] J Y Yan, L H Schwartz, B S Zhao. Semiautomatic segmentation of liver metastases on volumetric CT images, Medical Physics, 2015, 42(11): 6283-6293.

[7] F Liu, B S Zhao, P K Kijewski, L Wang, H Lawrence. Liver segmentation for CT images using GVF snake, Medical Physics, 2005, 32(12): 3699-3706.

[8] C Y Xu, J L Prince. Snakes, shapes, and gradient vector flow, IEEE Transactions on Image Processing A Publication of the IEEE Signal Processing Society, 1998, 7(3): 359-369.

[9] L Massoptier, S Casciaro. A new fully automatic and robust algorithm for fast segmentation of liver tissue and tumors from CT scans, European Radiology, 2008, 18(8): 1658-1665.

[10] S Al-Shaikhli, M Y Yang, B Rosenhahn. Automatic 3D Liver Segmentation Using Sparse Representation of Global and Local Image Information via Level Set Formulation, Computer Science, 2015.

[11] J K Wang, Y Z Cheng, C Y Guo, Y D Wang, S Tamura. ShapeCintensity prior level set combining probabilistic atlas and probability map constrains for automatic liver segmentation from abdominal CT images, International Journal of Computer Assisted Radiology and Surgery, 2015, 11(5): 817-826. 
[12] Y Boykov, G Funka-Lea. Graph Cuts and Efficient N-D Image Segmentation, International Journal of Computer Vision, 2006, 70(2): 109-131.

[13] Y Boykov, M P Jolly. Interactive graph cuts for optimal boundary and region segmentation of objects in $n$-d images, Proceedings of the IEEE International Conference on Computer Vision, 2001: 105-112.

[14] C Y Li, X Y Wang, S Eberl, M Fulham, et al. A Likelihood and Local Constraint Level Set Model for Liver Tumor Segmentation from CT Volumes, IEEE Transactions on Biomedical Engineering, 2013, 60(10): 2967-2977.

[15] J L Peng, F F Dong, Y M Chen, D X Kong. A region-appearance-based adaptive variational model for $3 D$ liver segmentation, Medical Physics, 2014, 41(4): 043502.

[16] J L Peng, Y Wang, D X Kong. Liver segmentation with constrained convex variational model, Pattern Recognition Letters, 2014, 43(1): 81-88.

[17] A Ben-Cohen, I Diamant, E Klang, M Amitai, H Greenspan. Fully convolutional network for liver segmentation and lesions detection, Springer International Publishing, 2016, 10008: 77-85.

[18] P F Christ, M E A Elshaer, F Ettlinger, S Tatavarty, et al. Automatic Liver and Lesion Segmentation in CT Using Cascaded Fully Convolutional Neural Networks and 3D Conditional Random Fields, International Conference on Medical Image Computing and Computer-Assisted Intervention, Springer, Cham, 2016, 9901: 415-423.

[19] F Lu, F Wu, P J Hu, Z Y Peng, et al. Automatic 3D liver location and segmentation via convolutional neural networks and graph cut, International Journal of Computer Assisted Radiology and Surgery, 2016, 12(2): 171-182.

${ }^{1}$ School of Mathematical Science, Zhejiang University, Hangzhou 310058, China.

${ }^{2}$ Department of Radiology, Zhejiang University Hospital, Hangzhou 310058, China.

${ }^{3}$ Department of Radiology, The First Affiliated HospitalZhejiang University School of Medicine, Hangzhou 310003, China.

${ }^{4}$ School of Mechanical Engineering, Zhejiang University, Hangzhou 310058, -China.

Email: dxkong@zju.edu.cn 